\title{
Parameter Estimation for Stochastic Differential Equations Driven by Mixed Fractional Brownian Motion
}

\author{
Na Song and Zaiming Liu \\ School of Mathematics and Statistics, Central South University, Changsha, Hunan 410075, China \\ Correspondence should be addressed to Na Song; songna@csu.edu.cn
}

Received 28 April 2014; Revised 20 July 2014; Accepted 20 July 2014; Published 3 August 2014

Academic Editor: Ali H. Bhrawy

Copyright (C) 2014 N. Song and Z. Liu. This is an open access article distributed under the Creative Commons Attribution License, which permits unrestricted use, distribution, and reproduction in any medium, provided the original work is properly cited.

\begin{abstract}
We study the asymptotic properties of minimum distance estimator of drift parameter for a class of nonlinear scalar stochastic differential equations driven by mixed fractional Brownian motion. The consistency and limit distribution of this estimator are established as the diffusion coefficient tends to zero under some regularity conditions.
\end{abstract}

\section{Introduction}

Stochastic differential equations (SDEs) are a natural choice to model the time evolution of dynamic systems which are subject to random influences. For existence and uniqueness of solutions of finite dimensional stochastic differential equations and properties of stochastic integrals, we refer to [1-3] and the references therein.

It is natural that a model contains unknown parameters. The parametric estimation problems for diffusion processes satisfying SDEs driven by Brownian motion (hereafter Bm) have been studied earlier. For a more recent comprehensive discussion, we refer to $[4,5]$ and the references therein. In case of statistical inference for diffusion processes satisfying SDEs driven by a fractional Brownian motion (hereafter $\mathrm{fBm}$ ), substantial progress has been made in this direction; we refer to [6-9] for more details.

The mixed fractional Brownian motion (hereafter $\mathrm{mfBm}$ ) was introduced by Patrick [10] to present a stochastic model of the discounted stock price in some arbitrage-free and complete financial markets. As a result, in order to take into account long memory and exclude arbitrage, it is natural to use $\mathrm{mfBm}$ to replace the standard Brownian motion. Consequently, there has been a growing interest in parameter estimation for stochastic processes driven by $\mathrm{mfBm}$.

There are several heuristic methods available for use in case of SDEs driven by $\mathrm{mfBm}$, such as MLE, LSE, and sequential estimation. In the continuous case, since the MLE has desirable asymptotic properties of consistency, normality, and efficiency under broad conditions, perhaps the most direct method is the MLE. However, MLE has some shortcomings; MLE's calculation is often cumbersome as the expressions for MLE involve stochastic integrals which need good approximations for computational purposes. Moreover, the generally good asymptotic properties are not always satisfied in the discrete case. Paper [11] showed that the approach of estimating parameters of an Itô process by applying MLE to a discretization of the SDE does not yield consistent estimators. LSE is asymptotically equivalent to the MLE. It is well known that the sequential estimation methods might lead to equally efficient estimators from the process observed possibly over a shorter expected period of observation time. Although there exists a wide range of estimation techniques developed for the problem of parameters estimation for SDEs driven by $\mathrm{mfBm}$, we should choose a suitable estimation method.

Though $\mathrm{mfBm}$ has stationary self-similar increments, it does not have stationary increments and is not a Markov process. So, state-space models and Kalman filter estimators cannot be applied to the parameters of this process. Under the circumstances, in order to overcome those difficulties, the minimum distance approach is proposed.

The interest for this method of parametric estimation is that the minimum distance estimation (hereafter MDE) method has several features. It makes MDE an attractive method. From one part it is sometimes easy to calculate. 
On the other side this estimator is known to be consistent (see [12]) under some general conditions. Millar [13] studied a general framework for (MDE) of Hilbertian type and showed that the MDE is efficient and asymptotically normal in some situations. Furthermore, the MDE is a class of estimators that is automatically robust in the same sense (for more details see [14]), which is generically optimal according to some quantitative measure of robustness.

For the SDEs driven by Brownian motions, Kutoyants [15] and Kutoyants and Pilibossian [16] proved that $\varepsilon^{-1}\left(\theta_{\varepsilon}^{*}-\right.$ $\theta_{0}$ ) converge in probability to the random variable $\zeta_{T}$ with $L_{1}, L_{2}$ or supremum norm and he also proved that $\zeta_{T}$ is asymptotically normal when $T \rightarrow+\infty$ and $\theta_{0}>0$. Hénaff [17] established the same results in the general case of a norm in some Banach space of functions on $[0, T]$. For the SDEs driven by $\mathrm{fBm}$, Prakasa Rao [18] studied the minimum $L_{1}$-norm estimator $\theta_{\varepsilon}^{*}$ of the drift parameter of a fractional Ornstein-Uhlenbeck type process and proved that $\varepsilon^{-1}\left(\theta_{\varepsilon}^{*}-\theta\right)$ converges in probability under $\mathbb{P}_{\theta_{0}}$ to a random variable $\zeta$. Kouame et al. [19] studied asymptotic properties of minimum distance estimator of the parameter of stochastic process driven by a $\mathrm{fBm}$ as the diffusion coefficient tends to zero.

However, it appears that there are few works studying the estimators of $\mathrm{mfBm}$. Zili [20] obtained some general stochastic properties of the $\mathrm{mfBm}$ and treated the Hölder continuity of the sample paths and $\alpha$-differentiability of the trajectories of $\mathrm{mfBm}$. Miao [21] obtained the asymptotic properties of the minimum $L_{1}$-norm estimator of the $\mathrm{drift}$ parameter for a linear SDE driven by an $\mathrm{mfBm}$. Xiao et al. [22] studied the problem of estimating the parameters for the $\mathrm{mfBm}$ from discrete observations based on the MLE.

In present paper, our aim is to obtain the MDE of the drift parameter for a class of nonlinear scalar SDEs driven by $\mathrm{mfBm}$ and study the asymptotic properties of this estimator.

The remainder of this paper proceeds as follows. Section 2 starts with a short description of definition of $\mathrm{mfBm}$ and then provides some basic lemmas that will be used in the forthcoming sections. And we obtain the MDE of the drift parameter for a class of nonlinear scalar SDEs driven by $\mathrm{mfBm}$. In Section 3, we study the consistency of the above estimator. In Section 4, the limit distribution of this estimator is established as the diffusion coefficient tends to zero under some regularity conditions.

\section{Notation and Preliminaries}

Let $B_{t}^{H}:=\left\{B^{H}(t), 0 \leq t \leq T\right\}$ be a fractional Brownian motion defined on the probability space $\left(\Omega, \mathscr{F},\left\{\mathscr{F}_{t}\right\}_{t \geq 0}, \mathbb{P}\right)$ and $\left\{\mathscr{F}_{t}\right\}_{t \geq 0}$ is a filtration of $\sigma$-algebra of $\mathscr{F}$, where the usual conditions are satisfied; that is, $(\Omega, \mathscr{F}, \mathbb{P})$ is a complete probability space, $\mathscr{F}_{0}$ contains all $P$-null sets of $\mathscr{F}$, and, for each $t \geq 0, \mathscr{F}_{+}:=\bigcap_{s>t} \mathscr{F}_{s}=\mathscr{F}_{t}$.

A fractional Brownian motion $B_{t}^{H}$ of Hurst parameter $H \in(0,1)$ is a continuous and centered Gaussian process; that is, $E\left(B_{t}^{H}\right)=0$ for all $t \geq 0$, with covariance function

$$
E\left(B_{s}^{H} B_{t}^{H}\right)=\frac{1}{2}\left[s^{2 H}+t^{2 H}-|s-t|^{2 H}\right], \quad t \geq 0, s \geq 0
$$

From (1), it is easy to obtain that $E\left[B_{t}^{H}\right]^{2}=t^{2 H}, t \geq 0$, for all $H \in(0,1)$. Moreover, the $\mathrm{fBm} B_{t}^{H}$ reduces to a standard Brownian motion denoted by $B_{t}:=\{B(t), 0 \leq t \leq T\}$ for $H=1 / 2$.

The notation $\left\{X_{t}\right\} \stackrel{d}{=}\left\{Y_{t}\right\}$ means that the $\left\{X_{t}\right\}_{t \in \mathbb{R}_{+}}$and $\left\{Y_{t}\right\}_{t \in \mathbb{R}_{+}}$have the same law. Denote by $X_{t}^{*}$ the supremum process

$$
X_{t}^{*}=\sup _{s \leq t}\left|X_{s}\right|
$$

A standard $\mathrm{fBm} B_{t}^{H}$ has the following properties (for more details see [23], Page 5, Definition 1.1.1).

(1) $B_{t}^{H}$ has homogeneous increments; that is, $B_{s+t}^{H}-B_{t}^{H} \stackrel{d}{=}$ $B_{t}^{H}$ for $s, t \geq 0$.

(2) $B_{t}^{H}$ has continuous trajectories.

Let us take $a$ and $b$ which are two real constants such that $(a, b) \neq(0,0)$. By Patrick [10], we introduce the following.

Definition 1. A mixed fractional Brownian motion of parameters $a, b$, and $H$ is a process $M^{H}:=\left\{M_{t}^{H}(a, b) ; t \geq 0\right\}$ $=\left\{M_{t}^{H} ; t \geq 0\right\}$, defined on the probability space $(\Omega, \mathscr{F}$, $\left.\left\{\mathscr{F}_{t}\right\}_{t \geq 0}, \mathbb{P}\right)$ by

$$
M_{t}^{H}=M_{t}^{H}(a, b):=a B_{t}+b B_{t}^{H}, \quad \forall t \in \mathbb{R}_{+} .
$$

Remark 2. From Zili [20], we know that $\mathrm{mfBm}$ is a mixedself-similar process:

$$
M_{h t}^{H}(a, b) \stackrel{d}{=}\left\{M_{t}^{H}\left(a h^{1 / 2}, b h^{H}\right)\right\},
$$

where $h>0$ is a constant. Furthermore it follows for the supremum process $M^{*}$ that

$$
M_{h t}^{H *}(a, b) \stackrel{d}{=}\left\{M_{t}^{H *}\left(a h^{1 / 2}, b h^{H}\right)\right\} .
$$

By using the self-similarity of $\mathrm{mfBm}$, we obtain the following lemma.

Lemma 3. Let $T>0$ be a constant and $M_{t}^{H}$ an $m f B m$ with parameter $a, b, H$; then for every $p>0$,

$$
\begin{aligned}
\mathbf{E}\left(M_{T}^{H *}(a, b)\right)^{p} & =\mathbf{E}\left(M_{1}^{H *}\left(a T^{1 / 2}, b T^{H}\right)\right)^{p} \\
& =\mathbf{E}\left(\sup _{t \leq 1}\left|a T^{1 / 2} B_{t}+b T^{H} B_{t}^{H}\right|\right)^{p} .
\end{aligned}
$$

The value of (6) is not known. However it is fortunate that we have the following two lemmas which give the bounds for the standard $\mathrm{Bm}$ and $\mathrm{fBm}$, respectively.

Lemma 4 (Burkholder-Davis-Gundy inequalities). For any stopping time $\tau$ with respect to the filtration generated by the Bm $B_{t}$, one has

$$
c(p) \mathbf{E}\left(\tau^{p / 2}\right) \leq \mathbf{E}\left(\left(B_{\tau}^{*}\right)^{p}\right) \leq C(p) \mathbf{E}\left(\tau^{p / 2}\right), \quad p>0,
$$

where the constants $c(p)$ and $C(p)>0$ depend only upon the parameter $p$. 
B-D-G have a long history and we cite only some works in this area. Maybe the first general results were due to Novikov $(p>1 / 2)$ and Burkholder (see $[24,25])$.

Lemma 5 (see [26]). Let $\tau$ be a stopping time with respect to the filtration generated by the $f B m B_{t}^{H}$. Then, for $H \in(1 / 2,1)$, one has

$$
\begin{array}{r}
c(p, H) \mathbf{E}\left(\tau^{p H}\right) \leq \mathbf{E}\left(\left(B_{\tau}^{H *}\right)^{p}\right) \leq C(p, H) \mathbf{E}\left(\tau^{p H}\right), \\
\forall p \geq 0,
\end{array}
$$

and, for $H \in(0,1 / 2)$, one has

$$
c(p, H) \mathbf{E}\left(\tau^{p H}\right) \leq \mathbf{E}\left(\left(B_{\tau}^{H *}\right)^{p}\right), \quad \forall p>0,
$$

where the constants $c(p, H)$ and $C(p, H)>0$ depend only upon the parameters $p, H$.

Lemma 6 (see [16, page 120]). Let $Z_{\varepsilon}(u), \varepsilon>0, u \in \mathbb{R}$, be a sequence of continuous functions and $Z_{0}(u)$ a convex function which admits a unique minimum $\xi \in \mathbb{R}$. Let $L_{\varepsilon}$, $\varepsilon>0$, be a sequence of positive numbers such that $L_{\varepsilon} \rightarrow+\infty$ as $\varepsilon \rightarrow 0$. We suppose that

$$
\lim _{\varepsilon \rightarrow 0} \sup _{|u|<L_{\varepsilon}}\left|Z_{\varepsilon}(u)-Z_{0}(u)\right|=0 .
$$

Then

$$
\lim _{\varepsilon \rightarrow 0} \arg \min _{|u|<L_{\varepsilon}} Z_{\varepsilon}(u)=\xi,
$$

where if there are several minima of $Z_{\varepsilon}$, we choose the arbitrary one.

Now we consider the parameter estimation problem for a class of nonlinear scalar mixed SDE in the following framework:

$$
d X_{t}=S_{t}(\theta, X) d t+\varepsilon d M_{t}^{H}, \quad X_{0}=x_{0}, 0 \leq t \leq T,
$$

where $S_{t}(\cdot, X)$ is a known measurable functional, the unknown drift parameter $\theta \in \Theta \subset \mathbb{R}$, and $\varepsilon>0$.

Denote $\theta_{0}$ by the true parameter of $\theta$. Let $\mathbb{P}_{\theta_{0}}^{(\varepsilon)}$ be the probability measure induced by the process $\left\{X_{t}\right\}$ and $x_{t}(\theta)$ the solution of the differential equation (12) with $\varepsilon=0$.

Assume that the trend functional of the above equation has the following form:

$$
S_{t}(\theta, X)=V\left(\theta, t, X_{t}\right)+\int_{0}^{t} K\left(\theta, t, s, X_{s}\right) d s,
$$

where $V\left(\theta, t, X_{t}\right)$ and $K\left(\theta, t, s, X_{s}\right)$ are two measurable functions.

The function $S_{t}(\theta, x)$ is measurable with respect to $(t, \theta)$ and, for any $\delta>0$, denote

$$
g(\delta):=\inf _{\left|\theta-\theta_{0}\right|>\delta}\left\|x_{t}(\theta)-x_{t}\left(\theta_{0}\right)\right\|_{T} .
$$

Note that $g(\delta)>0$ for any $\delta>0$.
The $L_{p \geq 1}$ or supremum norm can be denoted by \|\|$_{T}$ and the $\operatorname{MDE} \theta_{\varepsilon}^{*}$ (see [15]) is defined by

$$
\theta_{\varepsilon}^{*}:=\arg \min _{\theta \in \Theta}\|X-x(\theta)\|_{T} .
$$

We also need the following additional conditions.

$\left(\mathscr{A}_{1}\right)$ The functional $S_{t}(\theta, \cdot)$ is measurable and nonanticipative and satisfies the following inequalities: for all $t \in[0, T]$ and $X, Y \in \mathscr{C}[0, T]$,

$$
\left|S_{t}(\theta, X)-S_{t}(\theta, Y)\right| \leq L_{1} \int_{0}^{t}\left|X_{s}-Y_{s}\right| d s+L_{2}\left|X_{t}-Y_{t}\right|,
$$

$$
\left|S_{t}(\theta, X)\right| \leq L_{1} \int_{0}^{t}\left(1+\left|X_{s}\right|\right) d s+L_{2}\left(1+\left|X_{t}\right|\right) \text {, }
$$

where $L_{1}, L_{2}$ are positive constants.

$\left(\mathscr{A}_{2}\right)$ The measurable functions $V\left(\theta, t, X_{t}\right)$ and $K(\theta, t$, $\left.s, X_{s}\right)$ are continuously twice differentiable in $\theta$ and $x$.

$\left(\mathscr{A}_{3}\right)$ Suppose that

$$
\inf _{\theta \in \Theta}\|\dot{x}(\theta)\|_{T}>0
$$

and define by the random variable $\xi_{T}=\xi_{T}\left(\theta_{0}\right)$,

$$
\xi_{T}:=\arg \min _{u \in \mathbb{R}}\left\|x^{(1)}-u \dot{x}\left(\theta_{0}\right)\right\|_{T} .
$$

Denote $\dot{x}_{t}(\theta)$ by the derivatives of $x_{t}(\theta)$ with respect to $\theta$ and introduce a Gaussian process $x_{t}^{(1)}=x_{t}^{(1)}(\theta)$ which satisfies the equation

$$
\begin{aligned}
& d x_{t}^{(1)}= {\left[V_{x}^{\prime}\left(\theta, t, x_{t}\right) x_{t}^{(1)}\right.} \\
&\left.+\int_{0}^{t} K_{x}^{\prime}\left(\theta, t, s, x_{s}\right) x_{s}^{(1)} d s\right] d t+d M_{t}^{H}, \\
& x_{0}^{(1)}=0, \quad 0 \leq t \leq T,
\end{aligned}
$$

where $V_{x}^{\prime}$ and $K_{x}^{\prime}$ are the derivatives of $V(\vartheta, t, x)$ and $K(\vartheta, t$, $s, x)$ with respect to $x$ and $x_{t}^{(1)}$ is a derivative with probability one of $X_{t}$ with respect to $\varepsilon$ as $\varepsilon=0$.

In the paper, we will use $C$ to denote a generic constant which may vary from place to place.

\section{Consistency}

Theorem 7. If the above condition $\left(\mathscr{A}_{1}\right)$ is satisfied, then, for any $p>0$, there exist constants $C_{1}(p, H), C_{2}(p)>0$ such that, for every $\delta>0$,

$$
\begin{aligned}
\mathbf{P}_{\theta_{0}}^{(\varepsilon)}\left\{\left|\theta_{\varepsilon}^{*}-\theta_{0}\right|>\delta\right\} & \\
\leq 2^{p} C_{T}^{p} C^{p} \varepsilon^{p}(g(\delta))^{-p}( & C_{1}(p, H) T^{H p} \\
& \left.+C_{2}(p) T^{p / 2}\right)
\end{aligned}
$$


Proof. Condition $\left(\mathscr{A}_{1}\right)$ ensures the existence and uniqueness of a strong solution of (12). It is obvious that, with $\mathbb{P}_{\theta_{0}}^{(\varepsilon)}$ probability one,

$$
\left\|X_{t}-x_{t}\left(\theta_{0}\right)\right\|_{\infty} \leq C \varepsilon \sup _{0 \leq t \leq T}\left|M_{t}^{H}\right| .
$$

For any $\delta>0$,

$$
\begin{aligned}
& \mathbf{P}_{\theta_{0}}^{(\varepsilon)}\left\{\left|\theta_{\varepsilon}^{*}-\theta_{0}\right|>\delta\right\} \\
& \leq \mathbf{P}_{\theta_{0}}^{(\varepsilon)}\left\{\inf _{\left|\theta-\theta_{0}\right| \leq \delta}\left(\|X-x(\theta)\|_{T}\right) \leq \inf _{\left|\theta-\theta_{0}\right|>\delta}\left(\|X-x(\theta)\|_{T}\right)\right\} \\
& \leq \mathbf{P}_{\theta_{0}}^{(\varepsilon)}\left\{\left\|X-x\left(\theta_{0}\right)\right\|_{T}+\inf _{\left|\theta-\theta_{0}\right| \leq \delta}\left(\left\|x(\theta)-x\left(\theta_{0}\right)\right\|_{T}\right)\right. \\
& \left.\quad>\inf _{\left|\theta-\theta_{0}\right|>\delta}\left(\left\|x(\theta)-x\left(\theta_{0}\right)\right\|_{T}\right)-\left\|X-x\left(\theta_{0}\right)\right\|_{T}\right\} \\
& \leq \mathbf{P}_{\theta_{0}}^{(\varepsilon)}\left\{2\left\|X-x\left(\theta_{0}\right)\right\|_{T}>\inf _{\left|\theta-\theta_{0}\right|>\delta}\left(\left\|x(\theta)-x\left(\theta_{0}\right)\right\|_{T}\right)\right\} .
\end{aligned}
$$

By (21), we have

$$
\begin{aligned}
\mathbf{P}_{\theta_{0}}^{(\varepsilon)}\left\{\left|\theta_{\varepsilon}^{*}-\theta_{0}\right|>\delta\right\} & \leq \mathbf{P}_{\theta_{0}}^{(\varepsilon)}\left\{\|X-x(\theta)\|_{\infty}>\frac{g(\delta)}{2 C_{T}}\right\} \\
& \leq \mathbf{P}_{\theta_{0}}^{(\varepsilon)}\left\{\sup _{0 \leq t \leq T}\left|M_{t}^{H}\right|>\frac{g(\delta)}{2 \varepsilon C C_{T}}\right\} \\
& =\mathbf{P}_{\theta_{0}}^{(\varepsilon)}\left\{M_{T}^{H *}>\frac{g(\delta)}{2 \varepsilon C C_{T}}\right\} \\
& \leq \frac{\mathbb{E}\left(M_{T}^{H *}\right)^{p}}{\left(g(\delta) / 2 \varepsilon C C_{T}\right)^{p}} .
\end{aligned}
$$

Then using Lemmas 3,4 , and 5 , we obtain

$$
\begin{aligned}
\mathbf{P}_{\theta_{0}}^{(\varepsilon)} & \left\{\left|\theta_{\varepsilon}^{*}-\theta_{0}\right|>\delta\right\} \\
& \leq 2^{p} C_{T}^{p} C^{p} \varepsilon^{p}(g(\delta))^{-p}\left(C_{1}(p, H) T^{H p}+C_{2}(p) T^{p / 2}\right) \\
& =O\left((g(\delta))^{-p} \varepsilon^{p}\right) .
\end{aligned}
$$

Remark 8. As a consequence of the above theorem, we obtain that $\theta_{\varepsilon}^{*}$ converges in probability to $\theta_{0}$ under $P_{\theta_{0}}^{(\varepsilon)}$-measure as $\varepsilon \rightarrow 0$. Furthermore, the rate of convergence is of order $O\left(\varepsilon^{p}\right)$ for every $p>0$.

\section{Asymptotic Distribution}

Theorem 9. Under conditions $\left(\mathscr{A}_{2}\right)$ and $\left(\mathscr{A}_{3}\right)$, we have that the random variable $\varepsilon^{-1}\left(\theta_{\varepsilon}^{*}-\theta\right)$ converges in probability to a random variable whose probability distribution is the same as that of $\xi_{T}$ under $\mathbb{P}_{\theta_{0}}$.
Proof. Let $\nu=\nu_{\varepsilon}=\varepsilon \lambda_{\varepsilon} \rightarrow 0$ and $\lambda_{\varepsilon} \rightarrow+\infty$ as $\varepsilon \rightarrow 0$. Introduce the set

$$
\begin{aligned}
H_{0}=H_{0}(\nu)=\{\omega & : \inf _{\left|\theta-\theta_{0}\right|<\nu}\|X-x(\theta)\|_{T} \\
& \left.<\inf _{\left|\theta-\theta_{0}\right| \geq \nu}\|X-x(\theta)\|_{T}\right\} .
\end{aligned}
$$

For any $\omega \in H_{0}$, we have $\left|\theta_{\varepsilon}^{*}-\theta_{0}\right|<\nu_{\varepsilon}$.

In fact, according to (17) of condition $\left(\mathscr{A}_{3}\right)$ and Taylor formula, we obtain

$$
\begin{aligned}
g\left(v_{\varepsilon}\right) & =\inf _{|u|>v_{\varepsilon}}\left\|x\left(\theta_{0}+u\right)-x\left(\theta_{0}\right)\right\|_{T} \\
& =\inf _{|u|>v_{\varepsilon}}\left\|u \dot{x}\left(\widetilde{\theta}_{0}\right)\right\|_{T} \\
& =\left(\inf _{|u|>v_{\varepsilon}}|u|\right)\left\|\dot{x}\left(\widetilde{\theta}_{0}\right)\right\|_{T} \geq \kappa_{0} v_{\varepsilon},
\end{aligned}
$$

where $u \in \mathbb{R}, \kappa_{0}>0, \widetilde{\theta}_{0}=\theta_{0}+\alpha u$, and $0<\alpha<1$.

Then, by Theorem 7, we have

$$
\mathbf{P}_{\theta_{0}}^{(\varepsilon)}\left\{\left|\theta_{\varepsilon}^{*}-\theta_{0}\right|>v_{\varepsilon}\right\} \longrightarrow 0, \quad \varepsilon \longrightarrow 0 .
$$

Therefore, we just need to consider the behavior of the norm $\|X-x(\theta)\|_{T}$, for $\theta \in\left\{\theta:\left|\theta_{\varepsilon}^{*}-\theta_{0}\right|<\nu_{\varepsilon}\right\}$.

We have

$$
\begin{aligned}
\varepsilon^{-1}\|X-x(\theta)\|_{T} & =\left\|\frac{X-x\left(\theta_{0}\right)}{\varepsilon}-\frac{x(\theta)-x\left(\theta_{0}\right)}{\varepsilon}\right\|_{T} \\
& =\left\|x^{(1)}-u \dot{x}\left(\theta_{0}\right)+r+q\right\|_{T},
\end{aligned}
$$

where

$$
\begin{aligned}
\theta & =\theta_{0}+\varepsilon u, \quad|u|<\lambda_{\varepsilon}, \\
q_{t} & =\frac{X_{t}-x_{t}\left(\theta_{0}\right)}{\varepsilon}-x_{t}^{(1)}, \\
r_{t} & =\frac{X_{t}\left(\theta_{0}+\varepsilon u\right)-x_{t}\left(\theta_{0}\right)}{\varepsilon}-u \dot{x}\left(\theta_{0}\right) .
\end{aligned}
$$

Then using (12), (13), (19), and (21),

$\left|q_{t}\right|$

$$
\begin{gathered}
=\mid \int_{0}^{t}\left[\frac{S_{v}\left(\theta_{0}, X\right)-S_{v}\left(\theta_{0}, x\right)}{\varepsilon}-V_{x}^{\prime}\left(\theta_{0}, v, x_{v}\right) x_{v}^{(1)}\right. \\
\left.\quad-\int_{0}^{v} K_{x}^{\prime}\left(\theta_{0}, v, h, x_{h}\right) x_{h}^{(1)} d h\right] d v \mid \\
\leq \int_{0}^{t} \mid \frac{V\left(\theta_{0}, v, X_{v}\right)-V\left(\theta_{0}, v, x_{v}\right)}{\varepsilon} \\
-V_{x}^{\prime}\left(\theta_{0}, v, x_{v}\right) x_{v}^{(1)} \mid d v
\end{gathered}
$$




$$
\begin{aligned}
& +\int_{0}^{t} \int_{0}^{v} \mid \frac{K\left(\theta_{0}, v, h, X_{h}\right)-K\left(\theta_{0}, v, h, x_{h}\right)}{\varepsilon} \\
& -K_{x}^{\prime}\left(\theta_{0}, v, h, x_{h}\right) x_{h}^{(1)} \mid d h d v \\
& \leq \int_{0}^{t}\left|V_{x}^{\prime}\left(\theta_{0}, v, \widetilde{X}_{v}\right) \frac{X_{v}-x_{v}}{\varepsilon}-V_{x}^{\prime}\left(\theta_{0}, v, x_{v}\right) x_{v}^{(1)}\right| d v \\
& +\int_{0}^{t} \int_{0}^{v} \mid K_{x}^{\prime}\left(\theta_{0}, v, h, \widetilde{X}_{h}\right) \frac{X_{h}-x_{h}}{\varepsilon} \\
& -K_{x}^{\prime}\left(\theta_{0}, v, h, x_{h}\right) x_{h}^{(1)} \mid d h d v \\
& \leq \int_{0}^{t}\left|V_{x^{\prime}}\left(\theta_{0}, v, \widetilde{X}_{v}\right)\right|\left|\frac{X_{v}-x_{v}}{\varepsilon}-x_{v}^{(1)}\right| d v \\
& +\int_{0}^{t}\left|V_{x}^{\prime}\left(\theta_{0}, v, \widetilde{X}_{v}\right)-V_{x}^{\prime}\left(\theta_{0}, v, x_{v}\right)\right|\left|x_{v}^{(1)}\right| d v \\
& +\int_{0}^{t} \int_{0}^{v}\left|K_{x}^{\prime}\left(\theta_{0}, v, h, \widetilde{X}_{h}\right)\right|\left|\frac{X_{h}-x_{h}}{\varepsilon}-x_{h}^{(1)}\right| d h d v \\
& +\int_{0}^{t} \int_{0}^{v}\left|K_{x}^{\prime}\left(\theta_{0}, s, v, \widetilde{X}_{v}\right)-K_{x}^{\prime}\left(\theta_{0}, s, v, x_{v}\right)\right|\left|x_{v}^{(1)}\right| d h d v \\
& \leq C_{1} \int_{0}^{t}\left|q_{v}\right| d v+C_{2} \int_{0}^{t} \int_{0}^{v}\left|q_{h}\right| d h d v \\
& +\varepsilon C_{3} \sup _{0 \leq t \leq T}\left|M_{T}^{H}\right| \sup _{0 \leq t \leq T}\left|x_{t}^{(1)}\right|
\end{aligned}
$$

with some constants $C_{i}>0, i=1,2,3$. From (19), condition $\left(\mathscr{A}_{2}\right)$, and Lemma 4.13 (see [27]), we obtain

$$
\sup _{0 \leq t \leq T}\left|x_{t}^{(1)}\right| \leq C \sup _{0 \leq t \leq T}\left|M_{T}^{H}\right|
$$

then,

$$
\left|q_{t}\right| \leq C_{1} \int_{0}^{t}\left|q_{s}\right| d s+C_{2} \int_{0}^{t} \int_{0}^{s}\left|q_{v}\right| d v d s+\varepsilon C_{4} \sup _{0 \leq t \leq T}\left|M_{T}^{H}\right|^{2} .
$$

By using Lemma 4.13 once more, we get

$$
\sup _{0 \leq t \leq T}\left|q_{t}\right| \leq C \varepsilon \sup _{0 \leq t \leq T}\left|M_{T}^{H}\right|^{2}
$$

Now consider $r_{t}$; using the Taylor formula, we obtain

$$
\begin{aligned}
\sup _{|u|<\lambda_{\varepsilon}} \sup _{0 \leq t \leq T}\left|r_{t}\right| & =\sup _{|u|<\lambda_{\varepsilon}} \sup _{0 \leq t \leq T}\left|u\left(\dot{x}_{t}\left(\widehat{\theta}_{0}\right)-\dot{x}_{t}(\theta)\right)\right| \\
& \leq \sup _{|u|<\lambda_{\varepsilon}}|u| \sup _{0 \leq t \leq T}\left|\dot{x}_{t}\left(\widehat{\theta}_{0}\right)-\dot{x}_{t}(\theta)\right| \leq C \varepsilon \lambda_{\varepsilon}^{2},
\end{aligned}
$$

where $\widehat{\theta}_{0}=\theta_{0}+\beta \varepsilon u, \beta \in(0,1)$.

Introduce the functions

$$
Z_{\varepsilon}(u)=\left\|X-x\left(\theta_{0}+\varepsilon u\right)\right\|_{T}, \quad Z_{0}(u)=\left\|x^{(1)}-u \dot{x}\left(\theta_{0}\right)\right\|_{T} .
$$

By (33) and (34),

$$
\begin{aligned}
& \sup _{|u|<\lambda_{\varepsilon}}\left|Z_{\varepsilon}(u)-Z_{0}(u)\right| \\
& =\sup _{|u|<\lambda_{\varepsilon}}|| \frac{1}{\varepsilon}\left(X-x\left(\theta_{0}+\varepsilon u\right)\right)\left\|_{T}-\right\| x^{(1)}-u \dot{x}\left(\theta_{0}\right) \|_{T} \mid \\
& \leq \sup _{|u|<\lambda_{\varepsilon}}\left\|\frac{1}{\varepsilon}\left(X-x\left(\theta_{0}+\varepsilon u\right)\right)-\left(x^{(1)}-u \dot{x}\left(\theta_{0}\right)\right)\right\|_{T} \\
& \leq C_{T} \sup _{|u|<\lambda_{\varepsilon}}\left\|\frac{1}{\varepsilon}\left(X-x\left(\theta_{0}+\varepsilon u\right)\right)-\left(x^{(1)}-u \dot{x}\left(\theta_{0}\right)\right)\right\|_{\infty} \\
& \leq C_{T}\left(\sup _{0 \leq t \leq T}\left|q_{t}\right|+\sup _{|u|<\lambda_{\varepsilon}} \sup _{0 \leq t \leq T}\left|r_{t}\right|\right) \\
& \leq C \varepsilon \sup _{0 \leq t \leq T}\left|M_{T}^{H}\right|^{2}+C \varepsilon \lambda_{\varepsilon}^{2} .
\end{aligned}
$$

Therefore, if we choose $\lambda_{\varepsilon}$ such that $\varepsilon \lambda_{\varepsilon}^{2} \rightarrow 0$ when $\varepsilon \rightarrow 0$, then, with probability one, we have

$$
\sup _{|u|<\lambda_{\varepsilon}}\left|Z_{\varepsilon}(u)-Z_{0}(u)\right| \longrightarrow 0
$$

Using Lemma 6, we obtain the result. The proof completes.

\section{Conflict of Interests}

The authors declare that there is no conflict of interests regarding the publication of this paper.

\section{References}

[1] X. Mao, Stochastic Differential Equations and Applications, Elsevier, 2007.

[2] J. Bao and Z. Hou, "Existence of mild solutions to stochastic neutral partial functional differential equations with nonLipschitz coefficients," Computers \& Mathematics with Applications, vol. 59, no. 1, pp. 207-214, 2010.

[3] J. Bao, Z. Hou, and C. Yuan, "Stability in distribution of mild solutions to stochastic partial differential equations," Proceedings of the American Mathematical Society, vol. 138, no. 6, pp. 2169-2180, 2010.

[4] B. L. S. Prakasa Rao, Statistical Inference for Diffusion Type Processes, London, UK, Oxford University Press, 1999.

[5] Y. A. Kutoyants, Statistical Inference for Ergodic Diffusion Processes, Springer, London, UK, 2004.

[6] K. Bertin, S. Torres, and C. A. Tudor, "Drift parameter estimation in fractional diffusions driven by perturbed random walks," Statistics \& Probability Letters, vol. 81, no. 2, pp. 243-249, 2011.

[7] H. Yaozhong, N. David, X. Weilin, and Z. Weiguo, "Exact maximum likelihood estimator for drift fractional Brownian motion at discrete observation," Acta Mathematica Scientia, vol. 31, no. 5, pp. 1851-1859, 2011.

[8] Y. Hu and D. Nualart, "Parameter estimation for fractional Ornstein-Uhlenbeck processes," Statistics \& Probability Letters, vol. 80, no. 11-12, pp. 1030-1038, 2010. 
[9] B. L. S. P. Rao, Statistical Inference for Fractional Diffusion Processes, Wiley, 2011.

[10] C. Patrick, "Mixed fractional brownian motion," Bernoulli, vol. 7, no. 6, pp. 913-934, 2001.

[11] A. W. Lo, "Maximum likelihood estimation of generalized Itô processes with discretely sampled data," Econometric Theory, vol. 4, no. 2, pp. 231-247, 1988.

[12] W. C. Parr and W. R. Schucany, "Minimum distance estimation and components of goodness-of-fit statistics," Journal of the Royal Statistical Society B: Methodological, vol. 44, no. 2, pp. 178189, 1982.

[13] P. W. Millar, "A general approach to the optimality of minimum distance estimators," Transactions of the American Mathematical Society, vol. 286, no. 1, pp. 377-418, 1984.

[14] D. L. Donoho and R. C. Liu, "The "automatic" robustness of minimum distance functionals," The Annals of Statistics, vol. 16, no. 2, pp. 552-586, 1988.

[15] Y. A. Kutoyants, Identification of Dynamical Systems with Small Noise, Kluwer Academic, Dordrecht, The Netherlands, 1994.

[16] Y. Kutoyants and P. Pilibossian, "On minimum $L_{1}$-norm estimate of the parameter of the Ornstein-Uhlenbeck process," Statistics \& Probability Letters, vol. 20, no. 2, pp. 117-123, 1994.

[17] S. Hénaff, "Asymptotics of a minimum distance estimator of the parameter of the Ornstein-Uhlenbeck process," Comptes Rendus de l'Académie des Sciences I, vol. 325, no. 8, pp. 911-914, 1997.

[18] B. L. S. Prakasa Rao, "Minimum $l_{1}$-norm estimation for fractional Ornstein-Uhlenbeck type process," Theory of Probability and Mathematical Statistics, vol. 71, pp. 160-168, 2004.

[19] L. Kouame, M. N'Zi, and A. F. Yode, "Asymptotics of minimum distance estimator of the parameter of stochastic process driven by a fractional Brownian motion," Random Operators and Stochastic Equations, vol. 16, no. 4, pp. 399-407, 2008.

[20] M. Zili, "On the mixed fractional Brownian motion," Journal of Applied Mathematics and Stochastic Analysis, vol. 2006, Article ID 32435, 9 pages, 2006.

[21] Y. Miao, "Minimum $L_{1}$-norm estimation for mixed fractional Ornstein-Uhlenbeck type process," Acta Mathematica Vietnamica, vol. 35, no. 3, pp. 379-386, 2010.

[22] W.-L. Xiao, W. G. Z. Zhang, and X. L. Zhang, "Maximumlikelihood estimators in the mixed fractional Brownian motion," Statistics, vol. 45, no. 1, pp. 73-85, 2011.

[23] F. Biagini, Y. Hu, B. Øksendal, and T. Zhang, Stochastic Calculus for Fractional Brownian Motion and Applications, Springer, New York, NY, USA, 2008.

[24] A. A. Novikov, "The moment inequalities for stochastic integrals," Theory of Probability Its Applications, vol. 16, no. 3, pp. 538-541, 1971.

[25] D. L. Burkholder, "Distribution function inequalities for martingales," The Annals of Probability, vol. 16, no. 3, pp. 19-42, 1973.

[26] A. A. Novikov and E. Valkeila, "On some maximal inequalities for fractional brownian motions," Statistics \& Probability Letters, vol. 44, no. 1, pp. 47-54, 1999.

[27] Y. A. Kutoyants, Parameter Estimation for Stochastic Processes, Heldermann, Berlin, Germany, 1984. 


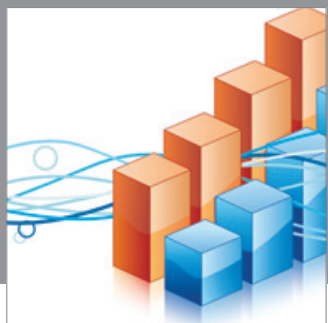

Advances in

Operations Research

mansans

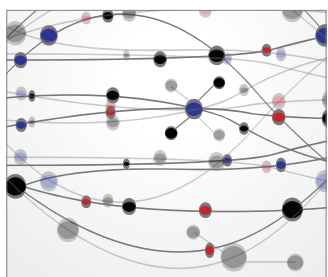

The Scientific World Journal
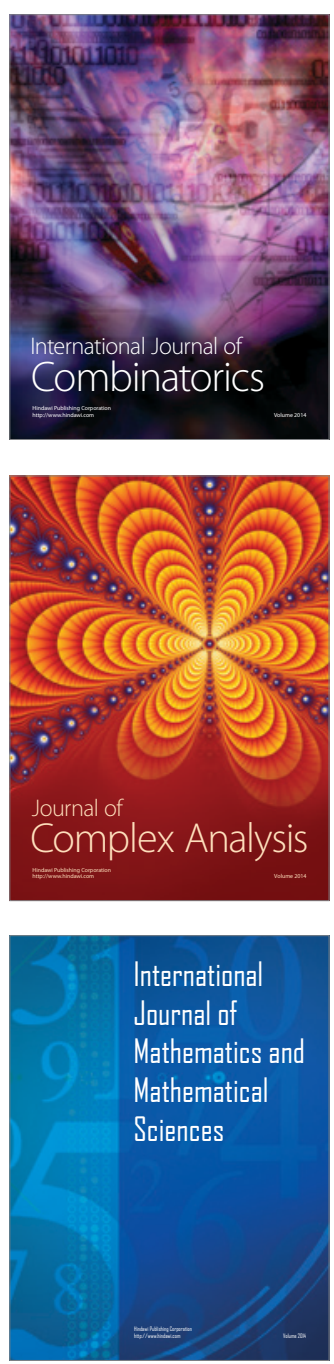
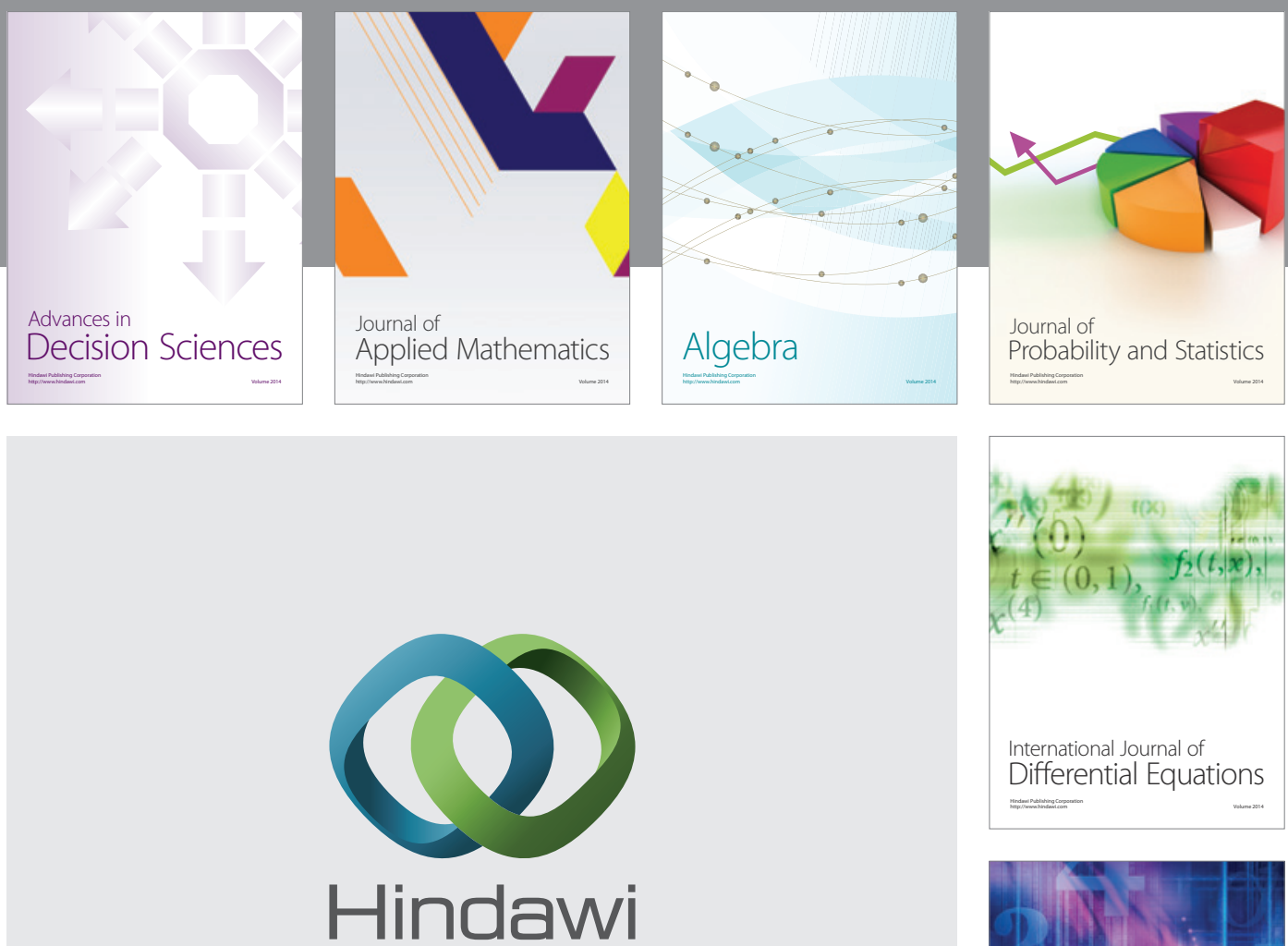

Submit your manuscripts at http://www.hindawi.com
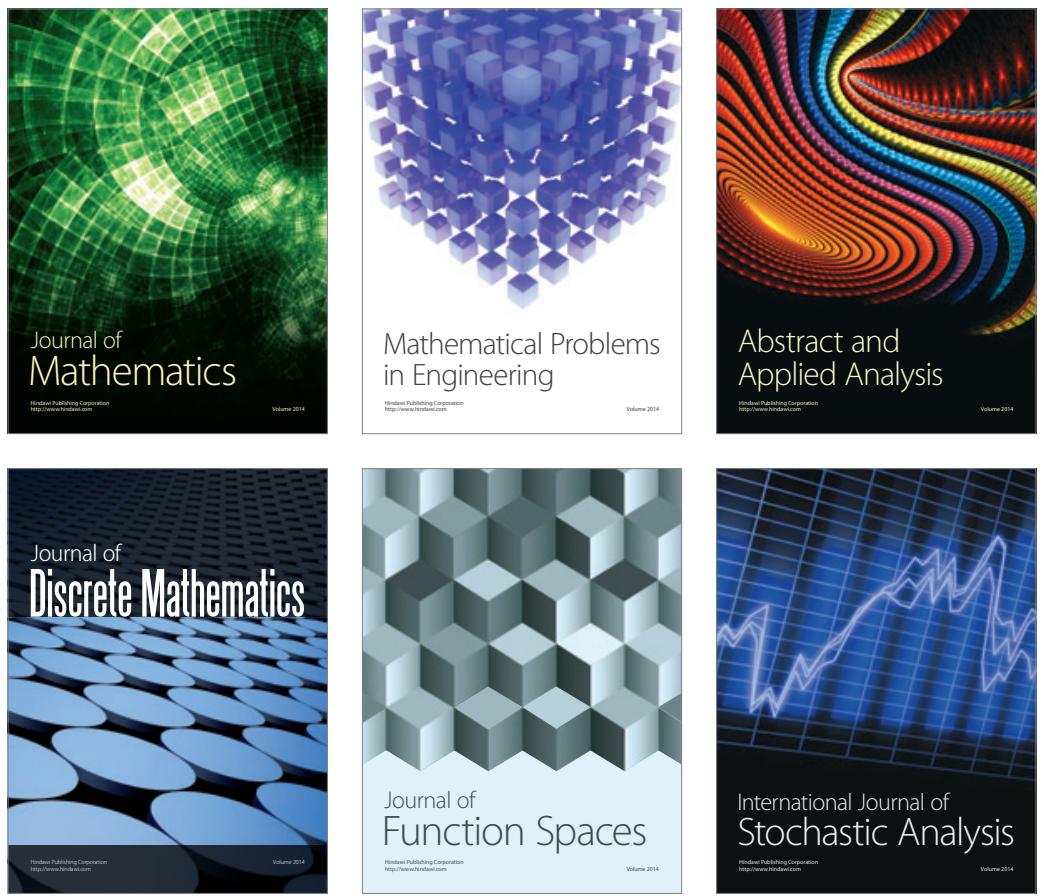

Journal of

Function Spaces

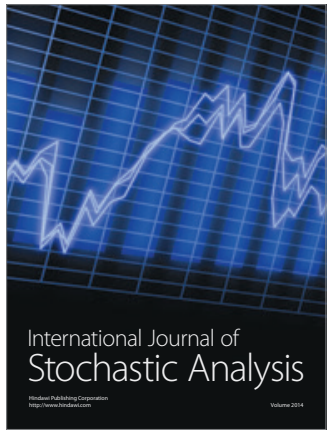

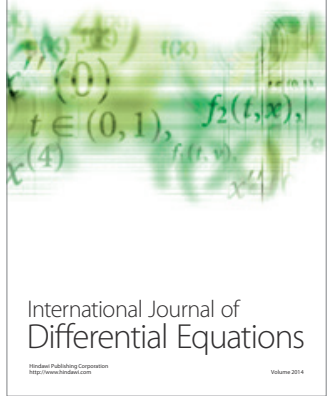
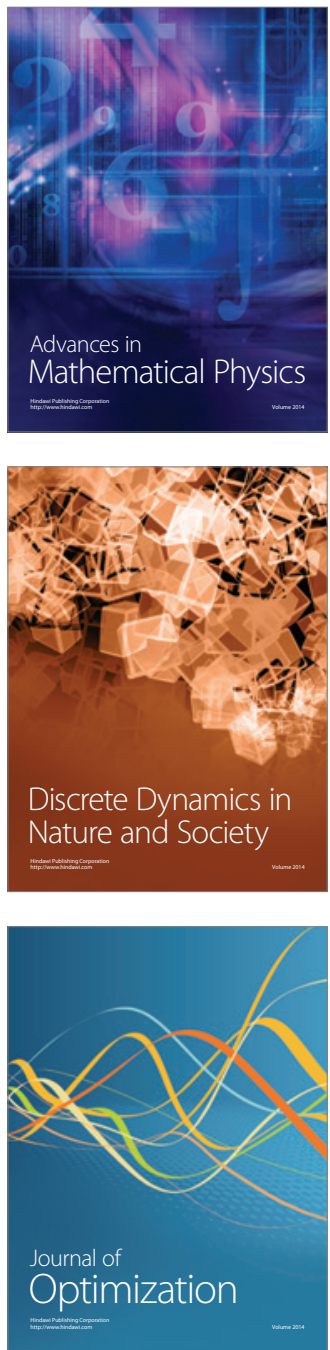